\title{
Processing visual ambiguity in fractal patterns: Pareidolia as a sign of creativity
}

Authors:

Antoine Bellemare Pepin ',2, Yann Harel ', Jordan O’Byrne ', Geneviève Mageau ', Arne Dietrich ${ }^{3}$, Karim Jerbi

Affiliations:

1. Computational and Cognitive Neuroscience Lab (CoCo Lab), Department of Psychology, Université de Montréal, Montréal, Canada

2. Department of Music, Concordia University, Montréal, Canada

3. Department of Psychology, American University of Beirut, Beirut 1107-2020, Lebanon

${ }^{*}$ Corresponding author: Antoine Bellemare Pepin

Email: antoine.bellemare9@gmail.com

Keywords: creativity, visual perception, pareidolia, fractal dimension, divergent perception 


\begin{abstract}
Creativity is a highly sought after and multifaceted skill. Unfortunately, we only have a loose grasp on its cognitive underpinnings. Empirical research typically probes creativity by estimating the potential for problem solving and novel idea generation, a process known as "divergent thinking". Here, by contrast, we examine creativity through the lens of perceptual abilities. In particular, we ask whether creative individuals are better at perceiving recognizable forms in noisy or ambiguous stimuli, a phenomenon known as pareidolia. To this end, we designed a visual perception task in which 50 participants, with various levels of creativity, were presented with ambiguous stimuli and asked to identify as many recognizable forms as possible. The stimuli consisted of cloud-like images with various levels of complexity, which we controlled by manipulating fractal dimension (FD) and contrast level. We found that pareidolic perceptions arise more often and more rapidly in individuals that are more creative. Furthermore, the emergence of pareidolia in individuals with lower creativity scores was more restricted to images with a narrow range of FD values, suggesting a wider repertoire for perceptual abilities in creative individuals. Our findings suggest that pareidolia may be used as a perceptual proxy of idea generation abilities, a key component of creative behavior. In sum, we extend the established body of work on divergent thinking, by introducing divergent perception as a complementary manifestation of the creative mind. These findings expand our understanding of the perception-creation link and open new paths in studying creative behavior in humans.
\end{abstract}

\title{
Significance Statement
}

Creativity is a highly valued and coveted skill. Unfortunately, its underlying cognitive processes are not yet fully understood. Typically, creativity is investigated by estimating the potential for creative problem solving and novel idea generation, a process known as "divergent thinking". Here, in an important departure from previous work, we introduce "divergent perception" as a new cognitive marker and predictor of creativity. Specifically, we show that the ability to perceive objects in ambiguous fractal stimuli, a phenomenon known as pareidolia, occurs more often and more rapidly in creative individuals. By demonstrating a tight link between perceptual and creative abilities, our findings open new paths to study, measure and possibly stimulate creative behavior in humans. 


\section{Introduction}

Creativity is a cornerstone of human evolution. It allows us to adapt to our environment and transform it. A widely accepted definition of creativity is the ability to produce work that is both novel (i.e., original, unexpected) and useful (i.e. adaptive given task constraints) (1), though it has been argued that this definition does not capture the full breadth and multiple facets of creativity (2-5). Additionally, although there have been many attempts to characterize the neural mechanisms underlying creativity (6-9), no consensus has yet emerged $(10,11)$. To date, most of the empirical research on creativity has focused on the concept of divergent thinking (DT; 12, 13), defined as the ability to generate multiple solutions to an open-ended problem $(12,14)$. The most widely used measure of creativity, the Torrance Tests of Creative Thinking (TTCT), consists mostly of DT tests (15). Two major problems arise when claiming that DT tests measure creativity per se $(16,17)$. First, it has been argued that the opposite - convergent thinking - can also produce creative ideas (18). Second, DT is a compound construct, which gathers multiple facets and mental processes within a single measure (19). These problems invite caution when approaching creativity as a monolithic entity (16) and beckon for complementary ways of operationalizing this multifaceted concept. Many accounts of creativity focus on cognitive processes, i.e. thinking differently. In this paper, we instead examine whether highly creative individuals differ in their perceptual processes, i.e. seeing differently.

It has recently been proposed that our ability to create depends heavily on our ability to perceive and model the external world (20). Indeed, creative individuals seem to process external sensory stimuli differently $(21,22)$, in that they will tend to more easily connect unrelated elements together. Accordingly, researchers have begun to investigate creativity through the lens of embodied cognition (23), which views action and perception as complementary. This approach emphasizes exploration and interactions with the physical environment as essential prerequisites for the emergence of cognition and therefore of creativity, attributing to attention the creative role of diversifying the field of experience and perceptual contents (24). A study on creative experience under the effect of psychedelics also distinguishes between creative performances, which can be recorded, and creative experiences, which are subjective and anchored in perception (25). This distinction between creative action (performance) and creative perception (experience) points to the importance of studying the phenomenology of creativity (26) complementarily to classical measures involving the production of creative artifacts. Finally, (27) suggested that creativity might emerge through confused perception (such as Beethoven's deafness), malfunctioning perception (such as psychotic symptoms), and intentional perception (expertise and use of analogical thinking). These cases of altered perception result from an increase in noise/uncertainty in the sensory signal whether through degradation of receptors, in the case of deafness or blindness, or through increased prediction errors and reduced latent inhibition, as it is the case in psychotic symptoms or psychedelic experiences. Together, these studies suggest that high-creative individuals have perceptual abilities that differ from low-creative individuals, and more specifically, that they might process ambiguous stimuli differently. However, to our knowledge, this has not yet been systematically investigated.

A natural approach to exploring inter-individual variability in processing ambiguous images is to exploit pareidolia, which is the experience of seeing meaningful patterns or connections in random stimuli (28), a fundamental aspect of human perception (29). Interestingly, pareidolia is thought to be an adaptive skill, as it may have helped early hominids to detect threats in complex sensory environments $(30,31)$. At the root of pareidolia is a concept called aberrant salience, which corresponds to an altered attentional state leading to a failed suppression of irrelevant or familiar information $(32,33)$. This decrease of latent inhibition may facilitate the emergence of pareidolic perceptions through concomitant complexification of sensory data and increases in top-down modulations. Pareidolia proneness is associated with schizophrenia symptoms, such as delusional thinking, paranoia and hallucinations (34-36). In a non-clinical population, a common example of pareidolia is the perception of meaningful objects 
in clouds. Clouds are complex visual stimuli which exhibit inherently unpredictable structures, making them ambiguous by nature. This ambiguity can be perceived in one of two ways. First, the brain can perceive it as noise with no relevant information and attribute the label cloud to the perceived image. In the second pareidolic instance, the brain makes an association between random features of the cloud and a known object, resulting in the perception of a meaningful object in noise such as a cat or a heart. However, it is important to note that this perceived cat or heart does not perfectly match any one previously perceived exemplar, but rather, it creatively emerges from the interaction of the semantic concept with the details of the fractal image. In short, "Pareidolia is a creative act because it is not about seeing things for what they are but seeing things for what they could be." (20).

Pareidolia has mostly been studied in the context of face detection (37-39), although more recent studies have also examined natural scenery images (40) and generative stimuli (41-43). Crucially, the empirical relation between creativity and pareidolia has received very little attention. A rare exception is an interesting study by Diana et al. (40) which suggests that fluency on a DT task significantly predicts fluency and originality in a Divergent Pareidolia Task. Pareidolia can be seen as a perceptual counterpart of DT, in that it relies on the possibility to find multiple solutions within a single problem space. The problem to be solved emerges from the ambiguity in the stimulus, and pareidolic perceptions are the multiple solutions to that problem. While DT probes creative thinking in the context of semantic problems resolved through conceptual expansion, divergent perception probes creative perception in the context of sensory problems resolved through pattern recognition. Pareidolia and creative thinking thus share the common fundamental process of relying on divergent processes to generate new ideas or percepts. In the context of DT, idea generation is measured in terms of fluency, flexibility and originality $(14,44)$. In divergent perception, percept generation can likewise be measured in terms of pareidolic fluency (finding multiple percepts in a unique stimulus) and flexibility (finding at least one percept in a wide range of stimuli).

Previous research indicates that the emergence of pareidolia depends on image properties including contrast and fractality. These properties are therefore likely to moderate the putative relation between the observer's creativity and their tendency to experience pareidolia. Image fractality can be quantified by its fractal dimension $(F D)$, a measure of the signal's self-similarity when observed at different magnifications. One of the first studies to assess the effect of $F D$ on pareidolia reported a relation between the $F D$ of generative cloud images and the prevalence of pareidolia (41). The authors presented four images simultaneously to participants and asked them to indicate the image in which a recognizable object was first detected. Although this method is useful to determine the $F D$ that preferably elicits spontaneous pareidolia, it does not speak to the systematic relations between fractality and pareidolia. Moreover, no statistical analysis was conducted on this dataset. A second study (43) investigated the relation between pareidolia and $F D$ by exposing 23 participants to a set of 24 images with $F D$ ranging from 1.05 to 1.95. Participants were asked to report the number of percepts elicited by each stimulus. Results indicated that lower FD stimuli elicited significantly more pareidolic percepts. While this study has the advantage of systematically investigating the relation between $F D$ and pareidolia, it is limited by a small sample size and a small number of stimuli. In the same vein, (42) demonstrated that object pareidolia is more diverse and occurred faster for $F D$ values close to 1.3. However, the number of stimuli used in the experiment was limited to 4 per $F D$, for a total of 16 , which may limit statistical inferences. Interestingly, the $F D$ that tends to facilitate pareidolia $(F D=1.3)$ has also been associated with the perception of beauty and aesthetic preference (45-47), both in synthetic noise images and works of art (48), suggesting a functional link between pareidolia and aesthetics.. Coherently, (49) developed a fluency model suggesting that mid-FDs (1.3-1.5) optimize both the observer's capacities of pattern recognition and the emergence of aesthetic experience. Individual differences between preferred patterns in a range of $F D$ have been systematically investigated by (50), who report that $90 \%$ of individuals can be classified as either preferring low, intermediate or high FDs, while no specific link between these profiles and creativity has been investigated. These individual differences in susceptibility to pareidolia across 
different FDs suggest that the modulation of image $F D$ offers a means to experimentally manipulate pareidolia in a laboratory setting.

Here, we set out to investigate the link between pareidolic perception and creative experience, as well as the moderating role of situational factors therein. To this end, we developed a novel pareidolia paradigm where fifty participants with various levels of creativity viewed a wide range of synthetic cloud-like images. Importantly, we generated the stimuli by manipulation of fractal dimension and contrast, and predicted that pareidolia would occur preferentially at intermediate $F D$ levels (close to 1.3) congruently with previous research (42) and that higher contrast would facilitate pareidolia. In a first step, we sought to validate the presence of a relationship between pareidolia and two key properties of the generated stimuli ( $F D$ and contrast). We then proceeded with our main objectives and hypothesized that creativity would be positively correlated with pareidolia. In terms of moderation, we expected that $F D$ and contrast level would moderate the relation between creativity and pareidolia. Specifically, we predicted that differences between low- and high-creatives would be amplified for optimal settings of $F D$ and contrast.

Creativity was primarily measured using the Experience of Creativity Questionnaire (ECQ; 51), which focuses on the phenomenological dimension of creativity and is designed to measure individual differences in the intensity of the creative experience as well as the depth of immersion when engaging in creative processes. This approach to assessing creativity is appealing for two main reasons: First, by being non-domain-specific, the ECQ can capture creativity irrespective of whether individuals engage in the production of creative artifacts or other classical artistic practices. Second, by focusing on the phenomenological aspect of the creative process, the ECQ captures the perceptual components of the creative experience, which according to our hypothesis, would correlate with pareidolia. This said, because the use of self-reported creativity measures has its limitations, we also administered a complementary creativity test that measures divergent thinking. For this, we used the recently proposed Divergent Association Task (DAT; 52), which requires the participant to find words that are the most semantically distant from one another. The associated creativity score is then computed automatically by estimating the mean semantic distance between the proposed words. Importantly, the DAT was shown to correlate with performance on two widely used creativity measures: the Alternative Uses Task and the Bridge-the-Associative-Gap Task. As a result, the use of the ECQ and DAT in the present study allows us to probe the relationship between self-reported creativity, divergent thinking and pareidolia.

\section{Results}

All participants viewed 360 visual fractal stimuli and were instructed to identify as many percepts as possible in each image during the 8-second presentation. The stimuli consisted of 3 levels of contrast and 12 levels of fractal dimensions ranging from 0.8 to 1.9 (Fig.1). Reaction times were measured as the latency of the button press indicating the emergence of the first pareidolic percept. We conducted an image-based analysis of reported pareidolia to address the potential issue of response bias. If the responses of the participants (i.e. pareidolia occurrence and number of percepts) were random and unrelated to pareidolia occurrence, we'd expect the mean pareidolia responses for the stimuli to be similar across all stimuli. Both for pareidolia occurrences and for the number of objects variables, we found that the response distributions across subjects were significantly different from the distributions of random behavioral responses (Fig. S2). These results indicate that the distribution of the original data significantly differs from that of randomly generated behavioral responses. 
Fractal Dimension
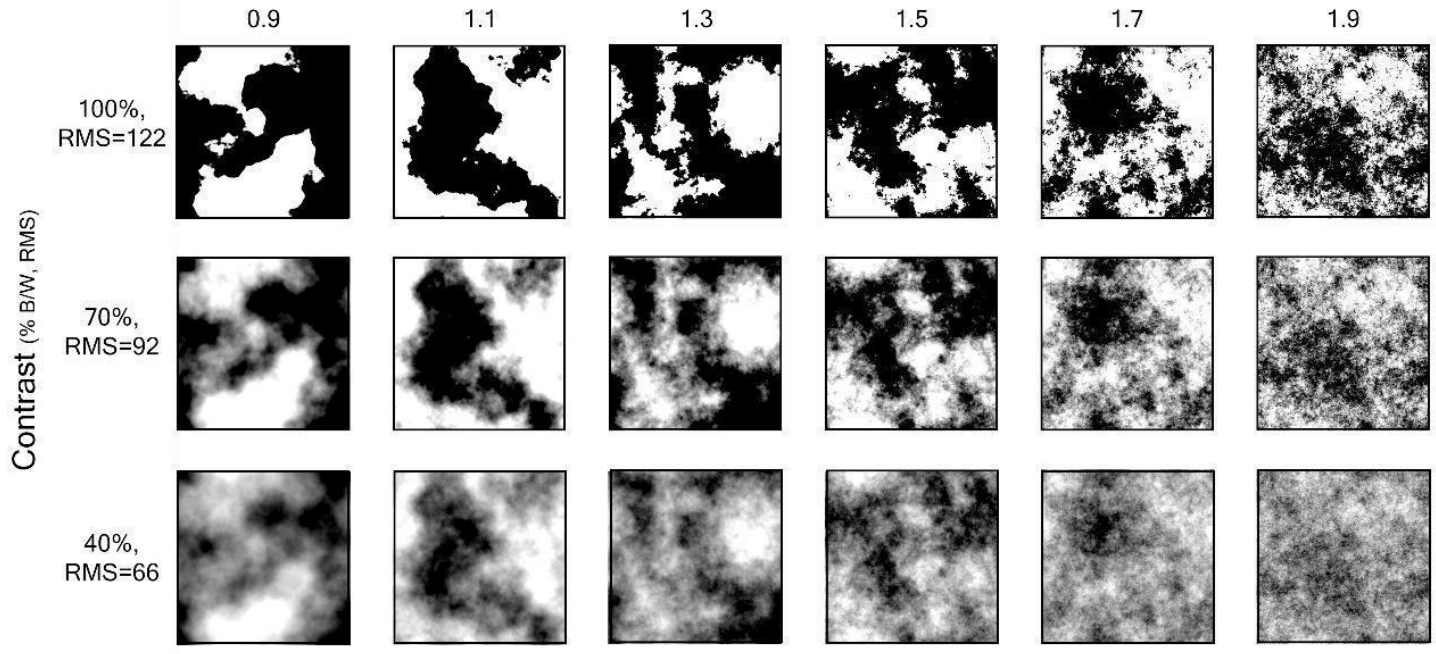

Figure 1. Stimuli generated with Fractional Brownian Motion algorithm. The X-axis represents the three levels of contrast, ranging from full black and white (left), to $20 \%$ of black and $20 \%$ of white (right). The Y-axis represents the fractal dimension of stimuli, ranging from 0.9 to 1.9.

\section{Effect of stimulus fractal dimension on pareidolia}

Considering the hypothesis that intermediate FDs (around 1.3) might facilitate pareidolia and given that the scatterplots showed clear inverted U-shapes, regressions were computed to model both the linear and the quadratic effect of $F D$ on each of the four dependent variables (see Fig. 2). We found significant quadratic relations between $F D$ and pareidolia occurrences (Par), $\mathrm{R}^{2}$ $=.91, F(2,9)=43.51, p<.001$, and number of objected $\left(N \_o b j\right), R^{2}=.86, F(2,9)=28.25, p<.001$, while the linear trend explained more variance than the quadratic trend for reaction time $(R T)$ and spontaneous pareidolia (Spont_par). These results indicate that low- to mid-FDs are associated with increased pareidolia, mid-FDs yield a higher number of pareidolic percepts, and low-FDs facilitate spontaneous pareidolia. 
a
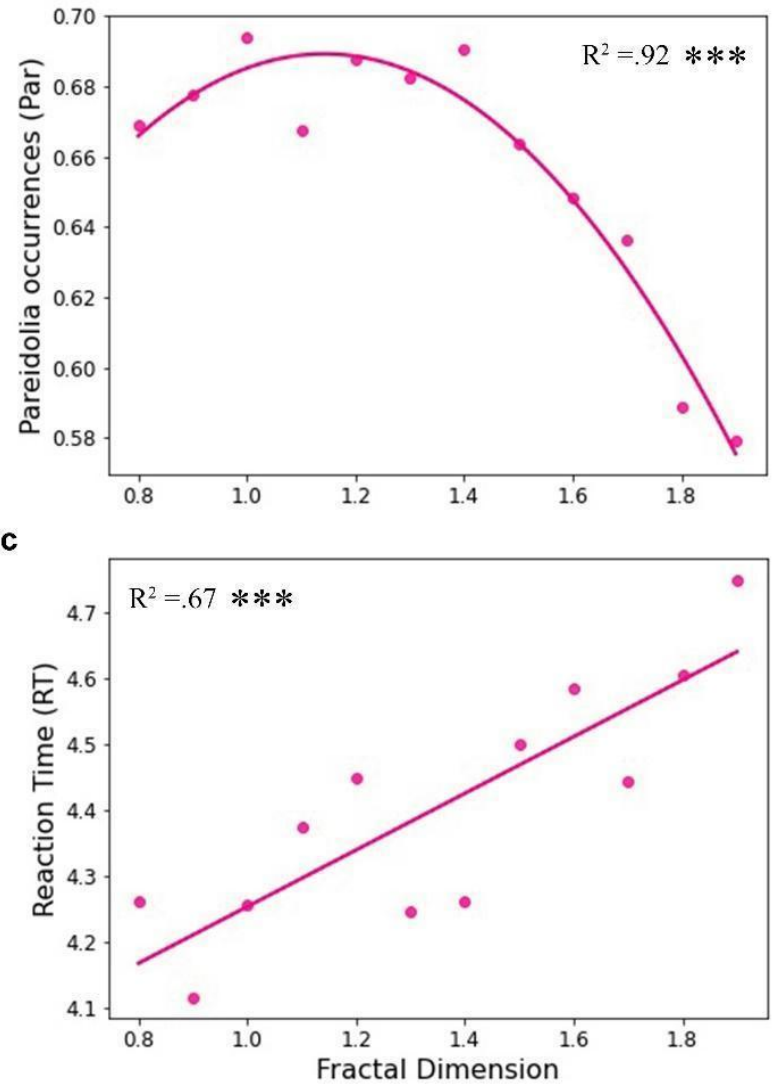

b

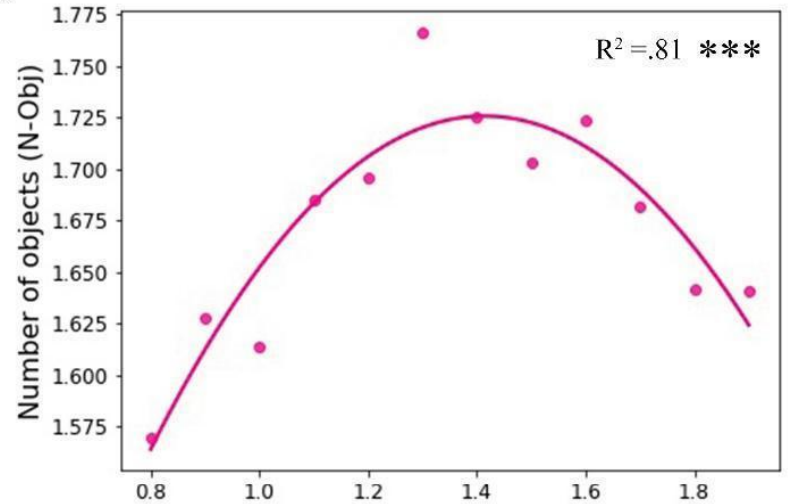

d

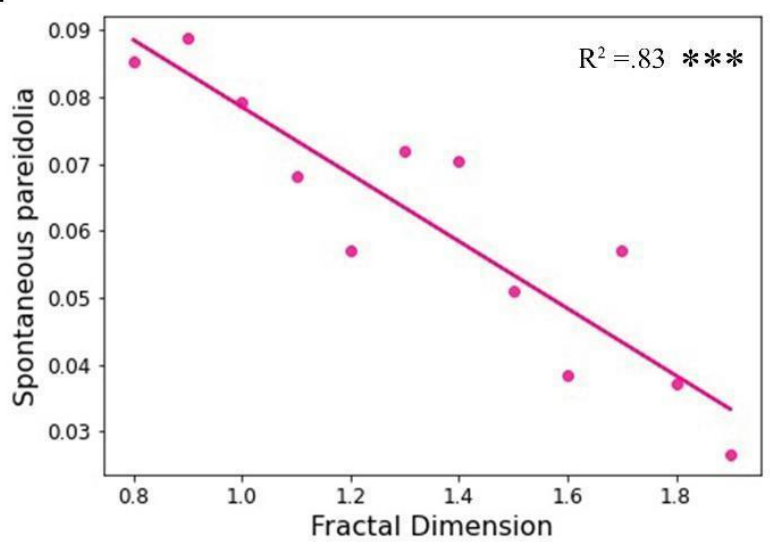

Figure 2. Pareidolia as a function of fractal dimension (FD). (a) Pareidolia occurrences (Par) as a function of $F D$. (b) Number of perceived objects for pareidolia trials as a function of $F D$. (c) Time before first pareidolic percept as a function of $F D$. (d) Proportion of pareidolia trials with reaction time shorter than 2 seconds. $\mathrm{R}^{2}$ corresponds to the adjusted coefficient of determination in the corresponding regression model. ${ }^{*}: p<.05 ;{ }^{* *}: p<.01 ;{ }^{* * *}: p<.001$

\section{Effect of image contrast on pareidolia}

To investigate the effect of contrast on pareidolia, we ran a repeated-measure ANOVA with a Greenhouse-Geisser correction on each of the four DVs. We found a statistically significant effect of contrast on the occurrence of pareidolia (Par), $F(1.65,74.03)=49.5, p<.001$, the number of pareidolic percepts $\left(N \_o b j\right), F(1.5,67.67)=81.94, p<.001$, reaction time $(R T), F(1.8$, $75.51)=11.46, p<.001$ ) and spontaneous pareidolia (Spont_par) $F(1.61,72.63)=8.78, p<.001$. Post hoc tests using the Bonferroni correction revealed that all contrast levels differed significantly $(p<.001)$ for Par and $N \_o b j$, while for $R T$ and Spont_par, only high-contrast images differed significantly from mid-contrast (RT: $p<.01$, Spont_par: $p=.02$ ) and low-contrast (both $p$ $<.01)$. These results indicate that high-contrast images facilitate both the flexibility and the fluency of pareidolia, as well as the speed of its emergence. 


\section{Relationship between creativity measures and pareidolia}

To test our main hypothesis that high-creatives experience pareidolia more often we computed the Spearman correlations between creativity (both self-reported and based on divergent thinking) and properties of pareidolic experience across participants. Spearman correlations were used because our measures of number of objects and spontaneous pareidolia were not normally distributed. More specifically, we assessed pairwise correlations between two measures of creativity (DAT and Crea_pheno) and four measures of pareidolia (Par, N_obj, Reaction Time and Spont_par), and controlled for multiple comparisons using the False Discovery Rate (FDR). The sample size for this correlation analysis was $n=42$ for correlations including DAT, $\mathrm{n}=48$ for correlations including $R T$ and Spont_par and $\mathrm{n}=50$ for all the other correlations. Figure 3 shows that Crea_pheno was significantly correlated with the four measures of pareidolia: $\operatorname{Par}(r(49)=0.55, p<.01), N$ obj $(r(49)=0.47, p<.05), R T(r(49)=-.36, p<.05)$, spont_par $(r(49)=.5, p<.01)$, while DAT scores were correlated with $\operatorname{par}(\mathrm{r}(41)=.5, p<.01)$, $N \_o b j(\mathrm{r}(41)=.37, p<.05), R T(\mathrm{r}(41)=.35, p<.05)$ and with Crea_pheno $(\mathrm{r}(41)=.33, p<.05)$.

To get a better understanding of the correlation between DAT (our measure of divergent thinking) and Crea_pheno (self-reported metric of creativity), we performed further correlation analysis between DAT scores and each of the two sub-dimensions of the Crea_pheno test: the first is related to one specific creative experience the participant identifies with (Crea pheno specific), and the second reflects the assessment of creative processes in a broader sense (Crea pheno general). The results are shown in Figure $\mathbf{4 a - b}$. Interestingly, this analysis revealed that divergent thinking (i.e. DAT) was significantly correlated with the general dimension of Crea_pheno $(\mathrm{r}(41)=.39, p=.01)$ but not with the sub-component that assesses a specific creative experience $(r(41)=.24, p=.13)$. The fact that both the DAT and Crea_pheno correlate strongly with pareidolia (Fig. 4c-d) and less strongly between each other may suggest that pareidolia is related to both the phenomenological aspects of the creative process and divergent thinking capacities.

Together, these results support the hypothesis that creative individuals are more prone to experience pareidolia. The main statistical models presented in the subsequent sections are built using Crea_pheno because this measure exhibited the strongest correlation with pareidolia and was available for all 50 participants (the DAT scores were only available in the 42 participants who were able to participate in the follow-up data collection). This said, the observed correlations between both types of creativity metrics and pareidolia will be useful for our interpretation and discussion. Results of the GLMM using DAT as a dependent variable are provided in supplementary material ; Table S1). 
Spearman's $r$

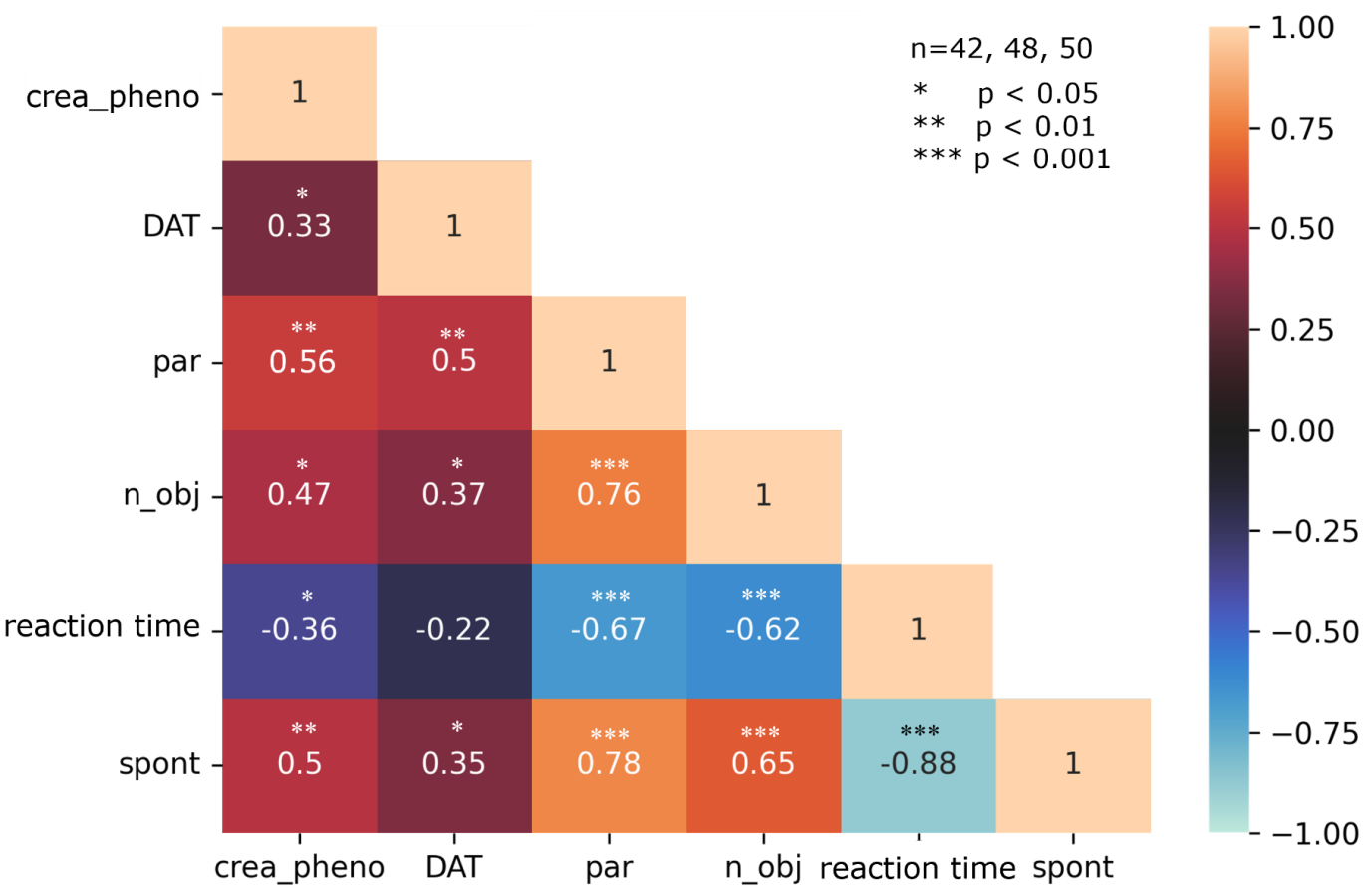

Figure 3. Correlation matrix of creativity measures and pareidolia. Par: Pareidolia occurrences, $\mathrm{N}$ _obj: Number of objects, reaction time: Reaction Time, spont_par: Spontaneous pareidolia, crea_pheno: Creative phenomenology, DAT: Divergent Association Task. All analyses were performed using maximum sample size $\left(n=42\right.$ for DAT, $n=48$ for RT and Spont_par, and $n=50$ for the rest). Significance levels were *: $p<.05 ;{ }^{* *}: p<$ $.01 ;{ }^{* * *}: p<.001$. 

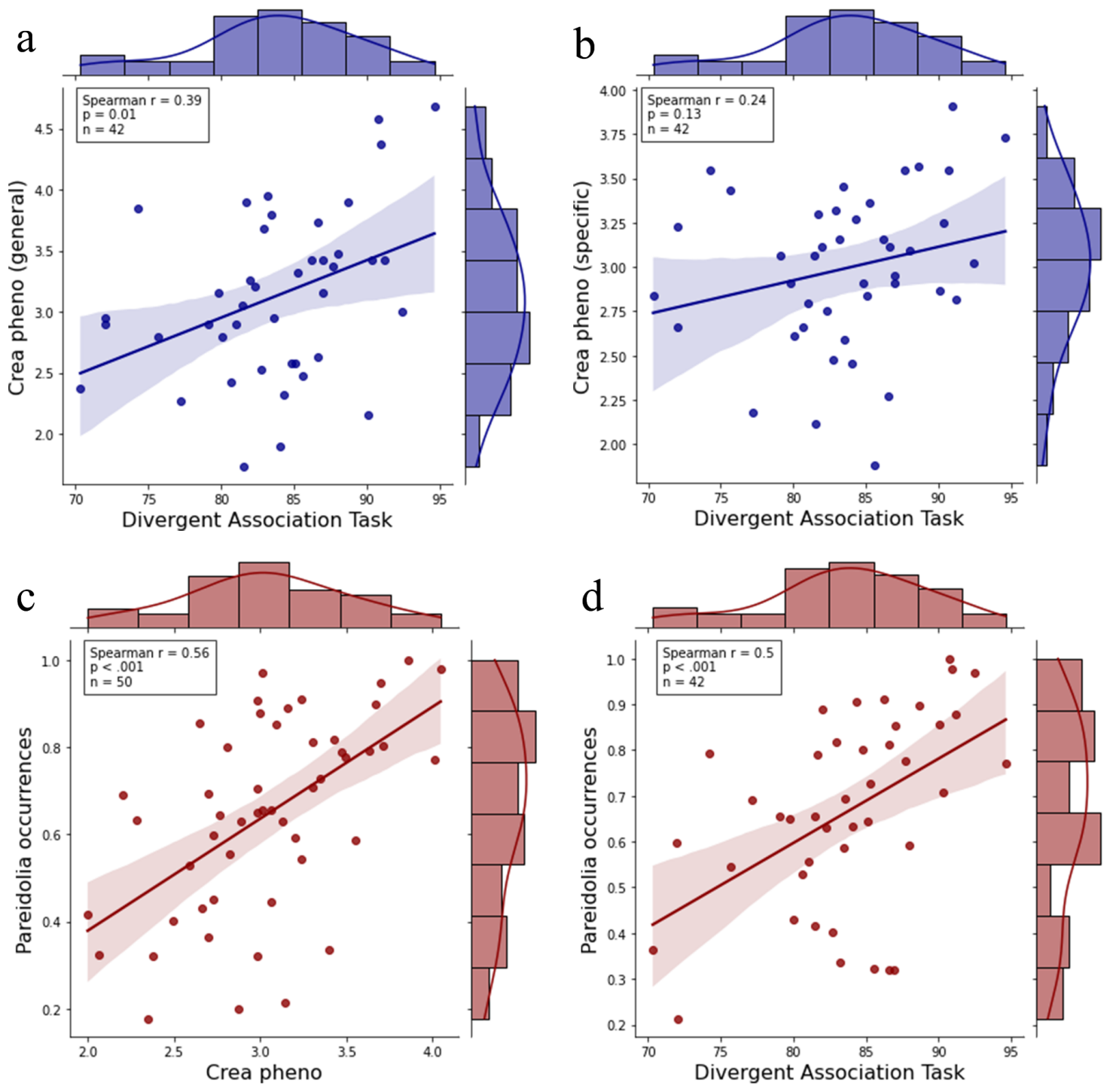

Figure 4. Regressions between self-reported creativity, divergent association and pareidolia occurrences (Par). (a) Significant correlation between the general dimension of Crea_pheno and DAT. (b) Non-significant correlation between the specific dimension of Crea_pheno and DAT. (c) Significant correlation between Crea_pheno and pareidolia occurrences. (d) Significant correlation between DAT and pareidolia occurrences. Pareidolia scores are averaged across all trials. 


\section{Interaction effects of creativity, fractal dimension and contrast on pareidolia}

We ran generalized linear mixed-effect models (GLMMs) that modelled the moderation effects of contrast and $F D$ on creativity in predicting pareidolia occurrences (Par), number of objects $\left(N \_o b j\right)$ and reaction time $(R T)$. A quadratic term $\left(F D^{2}\right)$ for both fixed and random effects of $F D$ was included in the model in order to account for its nonlinear relation with pareidolia. Contrast was only considered in the fixed effect structure, as it has been recommended that random variables must have more than 12 levels (53). The final model included all possible two-way interactions between creativity, $F D$ and contrast, as well as their three-way interaction. We ran the GLMMs with all the subjects, as well as without subjects with scores higher than 3SDs. We report the former case, while both cases result in the same significant effects.

The GLMM results in Table 1 and Figure 5 show that both $F D(p=.004)$ and contrast $(p$ $=.004$ ) interact significantly with creativity in predicting pareidolia occurrence, while the three-way interaction between $F D$, contrast and creativity was also significant. The two-way interactions validate the hypothesis that both $F D$ and contrast moderate the effect of creativity on pareidolia, while the significant effect of Crea_pheno alone reveals that creativity predicts pareidolia at average level of $F D$ and at high-contrast. The three-way interaction demonstrate that for high-contrast images, differences between low- and high-creatives are smaller for mid-FDs and larger for images of low- and high-FDs in predicting pareidolia

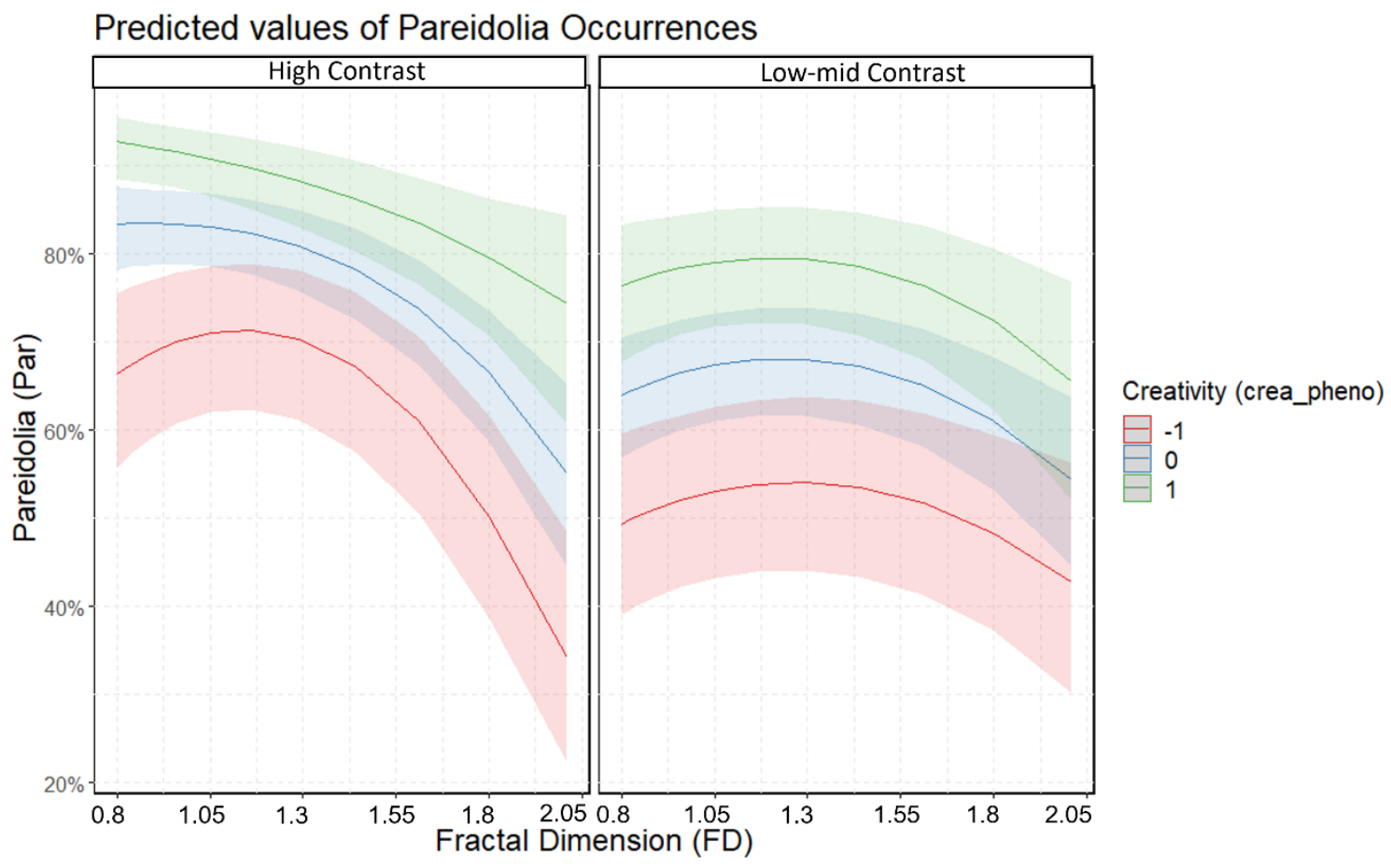

Figure 5. Predicted probabilities of the interaction between FD, Contrast and Creativity on pareidolia occurrences. Statistical values are in Table 1. 


\begin{tabular}{lrrrr}
\hline Fixed effects & Estimate & Std. Error & z value & p-value \\
\cline { 2 - 5 }$($ Intercept $)$ & 1.57 & 0.19 & 8.50 & $<0.001^{* * *}$ \\
$F D$ & 0.35 & 0.40 & 0.87 & 0.38 \\
$F D^{2}$ & -1.2 & 0.35 & -3.44 & $<0.001^{* * *}$ \\
Contrast & -0.98 & 0.11 & -8.97 & $<0.001^{* * *}$ \\
Creativity & 1.11 & 0.19 & 5.92 & $<0.001^{* * *}$ \\
$F D^{*}$ Creativity & -1.21 & 0.42 & -2.88 & $0.004^{* *}$ \\
$F D^{2 *}$ Creativity & 0.94 & 0.36 & 2.6 & $0.009^{* *}$ \\
\hline$F D^{*}$ Contrast & 0.45 & 0.44 & 1.43 & 0.31 \\
$F D^{2 *}$ Contrast & 0.29 & 0.38 & 0.77 & 0.44 \\
\hline Contrast ${ }^{*}$ Creativity & -0.33 & 0.12 & -2.86 & $0.004^{*}$ \\
$F D^{*}$ Creativity ${ }^{*}$ Contrast & 1.22 & 0.46 & 2.63 & $0.009^{* *}$ \\
$F D^{2 *}$ Creativity * Contrast & -1.02 & 0.40 & -2.57 & $0.01^{*}$ \\
\hline
\end{tabular}

Table 1. Moderation effect of $F D$ and Contrast on Creativity in predicting Pareidolia (Par). Number of participants $=50$, number of trials $=360$, total $N=18,000{ }^{*}: p<.05 ;{ }^{* *}: p<.01 ;{ }^{* * *}: p<.001$.

For the GLMM predicting $N \_o b j$, the moderation of stimulus properties on the relation between creativity and $N$ _obj was not significant. Thus, a model only containing the main effects was adopted. We found significant fixed effects for the two moderator variables, $F D(p<0.001)$, $F D^{2}(p<0.001)$, and contrast $(p<0.001)$, as well as for Crea_pheno $(p<0.001)$ (see Table. 2$)$.

\begin{tabular}{lrrrr}
\hline $\begin{array}{l}\text { Fixed } \\
\text { effects }\end{array}$ & Estimate & $\begin{array}{r}\text { Std. } \\
\text { Error }\end{array}$ & $\begin{array}{r}z \\
\text { value }\end{array}$ & p-value \\
\cline { 2 - 5 } (Intercept) & -0.07 & 0.09 & -0.81 & 0.42 \\
$F D$ & 0.90 & 0.18 & 5.04 & $<0.001^{* * *}$ \\
$F D^{2}$ & -0.82 & 0.16 & -4.99 & $<0.001^{* \star *}$ \\
Contrast & -0.33 & 0.02 & -16.92 & $<0.001^{* * *}$ \\
Creativity & 0.35 & 0.07 & 4.75 & $<0.001^{* \star *}$ \\
\hline
\end{tabular}

Table 2. Main effects of $F D$, Contrast and Creativity in predicting Number of Objects (N_obj).

Number of participants $=50$, number of trials $=360$, total $N=11,618{ }^{*}: p<.05 ;{ }^{* *}: p<.01{ }^{* * \star}: p<.001$. 
We conducted a third GLMM that predicts reaction time $(R T)$. This revealed significant fixed effects of Crea_pheno, FD and Contrast, and two-way interactions between contrast and FD $(p<.001)$ and between contrast and Crea_pheno (see Table. 3). The first interaction revealed that differences in RTs between high- vs. low-mid contrast were enhanced for low-FDs, indicating that high-contrast images increase the probability of shorter reaction time especially for images with lower fractal dimension. The second interaction revealed that Note that no GLMM was built to predict spontaneous pareidolia (Spont_par) since this variable was on the participant's level rather than on the observation's level.

\begin{tabular}{lrrrr}
\hline Fixed effects & Estimate & $\begin{array}{c}\text { Std. } \\
\text { Error }\end{array}$ & t value & p-value \\
\cline { 2 - 5 } (Intercept) & 1.38 & 0.03 & 53.67 & $<0.001^{* * *}$ \\
$F D$ & 0.15 & 0.23 & 6.55 & $<0.001^{* * *}$ \\
Contrast & 0.10 & 0.02 & 6.14 & $<0.001^{* * *}$ \\
Creativity & -0.1 & 0.03 & -3.72 & $<0.001^{* * *}$ \\
$F D^{*}$ Creativity & 0.02 & 0.02 & 0.89 & 0.37 \\
$F D^{*}$ Contrast & -0.08 & 0.03 & -3.21 & $0.001^{* *}$ \\
Contrast ${ }^{*}$ Creativity & 0.04 & 0.02 & 2.05 & $0.04^{*}$ \\
\hline$F D^{*}$ Creativity * & -0.01 & 0.03 & -0.45 & 0.65 \\
Contrast & & & & \\
\hline
\end{tabular}

Table 3. Moderation of $F D$ and Contrast on Creativity in predicting Reaction Time (RT).

Number of participants $=48$, number of trials $=360$, total $N=9,557{ }^{*}: p<.05 ;{ }^{* *}: p<.01 ;{ }^{* * *}: p<.001$.

We also conducted post-hoc Pearson correlation analyses to predict pareidolia occurrence (Par) from creativity scores at each level of $F D$ and for the two categories of contrast. False Discovery Rate (FDR) correction for multiple comparisons was applied. As shown in Table 4, for high-contrast images, our results show a larger effect of crea_pheno for low- and high-FD in predicting pareidolia occurrence, indicating that the difference between low- and high-creatives is smaller for mid-FDs. For low-to-mid-contrast images, the trend is less clearly defined.

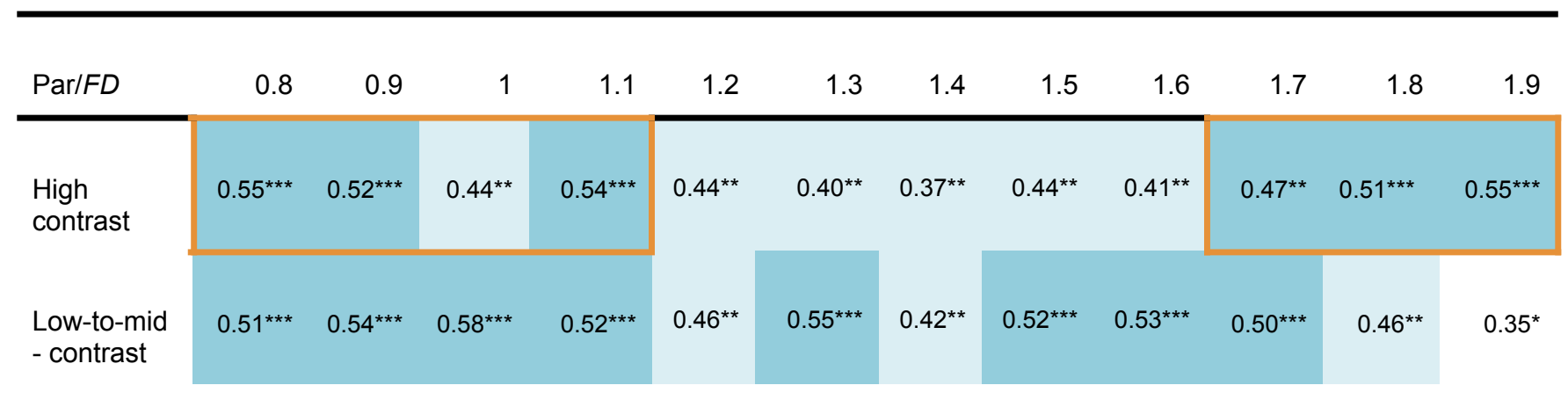

Table 4. Regression analyses for each Fractal Dimension $(F D)$ to predict pareidolia from creativity scores. Corrected $p$-values: ${ }^{*}: p<.05 ;{ }^{* *}: p<.01 ;{ }^{* * *}: p<.001 . n=50$ for each regression 


\section{Discussion}

In this study, we investigated the putative relationship between creativity and divergent perception. To do so, we implemented a novel pareidolia task in which participants were asked to identify recognizable forms in cloud-like images with different levels of fractality and contrast.

Typically, creativity is investigated by estimating the potential for creative problem solving and novel idea generation, a process known as divergent thinking. Here, in an important departure from previous work, we introduce divergent perception as a novel cognitive marker and predictor of creativity. Our results show that the perception of objects in ambiguous fractal stimuli (i.e. pareidolia) occurs more often and more rapidly in creative individuals. More specifically, linear mixed-effect modelling revealed that high-creative individuals are more flexible (wider range of optimal $F D s$ ), fluent (higher number of percepts) and faster in experiencing pareidolia. We also found that the association between creativity and pareidolia is stronger for high-contrast images with either low- or high-FD. Taken together, these results suggest that divergent perception captures a key cognitive feature of creativity, complementing established findings on divergent thinking. These results may have several future applications, in which pareidolic performance may be considered as a practical and easy-to-implement index of creativity or possibly a metric to monitor in the context of creativity training. In the following we will discuss our main observations reported in light of previous work.

\section{Pareidolia depends on image contrast and fractal dimension}

Quadratic regression analyses revealed that low- to mid-FDs maximize occurrences of pareidolia across trials, while mid-range FDs promote higher numbers of percepts during trials of pareidolia. This last result is congruent with (42) who reported enhanced pareidolia in images with $F D$ around 1.3. Consistently, quadratic regression analysis on reaction time indicates that pareidolia arises faster in low-FDs. Hence, pareidolia seems to be generally facilitated by stimuli of lower levels of inherent complexity, consistently with the findings of $(41,43)$, while mid- $F D$ images facilitate the experience of multiple pareidolic percepts. Our results showed that higher levels of contrast are associated with increased pareidolia occurrence and number of perceived objects, as well as shorter reaction time. This effect is consistent with the figure-ground segregation principle of Gestalt theory, which explains that as contrast increases, so does the perceptual saliency of what is perceived as the object compared to what is identified as the background (54). In the case of the pareidolia task, high contrast images seem to facilitate dissociation between black and white structures, leading to increased figure-ground segregation.

\section{Higher creativity is associated with higher propensity to pareidolia}

Our findings support the hypothesis that creativity is correlated with the propensity to pareidolia. In particular, the correlation analysis (Fig. 3) revealed that pareidolia occurrence is correlated both with phenomenological creativity and with divergent thinking abilities, measured via the Experience of Creativity Questionnaire (ECQ) and Divergent Association Task (DAT) metrics respectively. These results support the view that creativity, measured with two complementary tools, is predictive of pareidolia during perception of fractal visual noise. Moreover, the fact that the two measures were significantly correlated with pareidolia despite only being moderately correlated with each other points to the tempting hypothesis that pareidolia is associated with multiple components of the creative process.

The GLMM results further demonstrated the significant fixed effect of creativity on the occurrence of pareidolia, the number of pareidolic percepts and reaction time, indicating with more confidence that creative individuals experience pareidolia more often (for different stimuli), more rapidly, and that they perceive higher number of percepts when pareidolia occurs. These findings are in line with studies on bistable perception demonstrating that self-reported creative 
individuals are able to reverse the percept significantly more often (55-57), which requires the capacity to inhibit one percept over another. The role of (dis)inhibitory processes in creativity is not well established, and conflicting results indicate that both inhibitory (58) and disinhibitory (59) processes might participate in the emergence of creative behaviour. Other studies point to the role of cognitive flexibility in creative ideation $(60,61)$, while future studies are still required to investigate the relation between these processes and pareidolia.

Only very few studies have investigated the connections between pareidolia and creativity. One recent study (62) measured the impact of perceiving ambiguous stimuli on subsequent creative outcomes. Their results show that participants who passively looked at ambiguous figures, compared to non-ambiguous figures, scored higher on fluency, flexibility and originality in a subsequent Alternative Uses Task, and on creativity in a story generation task, thereby suggesting that processing ambiguous stimuli could have a beneficial priming effect on creative processes. Another recent study demonstrated that performances on both free association and divergent thinking tasks were predictive of pareidolic fluency and originality (40), also pointing to a functional role of pareidolia in creativity. With the present study, we incorporated a parametric manipulation of image complexity and showed that pareidolia in low-creative individuals exhibited a stronger dependence on stimulus properties, while divergent perception skills of high-creatives were less dependent on the physical properties of the stimulus.

\section{Higher creatives experience pareidolia across a larger repertoire of stimulus properties.}

Regression analyses revealed specific patterns of relation between creativity and pareidolia depending on the contrast level and the fractal dimension of the images. Analyzing high-contrast images, which previous analyses have shown to generally facilitate pareidolia more than low-mid-contrast images, we found that creativity predicts pareidolia specifically for low- and high-FD images. Two conclusions emerge from this result. First, it indicates that high-creatives seem to be more flexible in the way they integrate ambiguous information, as reflected by their ability to experience pareidolia in a wider range of FDs. Since divergent perception skills of high-creatives are less dependent on the physical properties of the stimulus, this would probably reflect a greater capacity to voluntarily produce the pareidolic effect, even under less favorable stimulation conditions. Their ease to experience pareidolia in high-FDs images is coherent with preliminary results showing that self-reported high-creative individuals prefer looking at images with higher FD (63), which seems to have been the case for Jackson Pollock, whose paintings show increasing FD throughout his lifetime (64). The ability of creative individuals to detect multiple percepts more easily in ambiguous visual stimuli might result from a tendency to depart from the propensity to automatize perception toward a single, most predictable percept. Even though automatization of perception might facilitate behavioral efficiency in most of our daily tasks, the present findings suggest that creative individuals might rely on defusing these perceptual habits to maximize novelty seeking and idea generation.

Second, our results suggest that mid-FDs (around 1.3) may facilitate pareidolia, notwithstanding the level of creativity. This effect is further illustrated by the significant quadratic relationship between $F D$ and pareidolia only for the low-creative group. These findings complement the studies of $(41,43)$, that showed a facilitation of pareidolia for images of $F D 1.3$, by indicating that this effect may be more predominant for low-creative individuals. Given that images of $F D 1.3$ have also been associated with perception of beauty (65), the present results suggest that stimuli perceived as aesthetic concomitantly facilitate the emergence of pareidolic percepts. This view is in line with theories of embodied cognition which posit that aesthetic quality is not a property of the stimulus, but an emergent phenomenon derived from the interaction between brain and stimulus (66). 


\section{Spontaneous and deliberate pareidolia}

The difference in reaction times as a function of creativity leads to the hypothesis that low- and high-creatives may rely on different perceptual strategies. One neuroscientific framework that may help to understand the mechanisms involved in pareidolia is the model proposed by Dietrich (17), which suggests a distinction between deliberate and spontaneous modes of creativity. The deliberate mode implies a conscious process of trial-and-error, which recruits a large amount of cognitive and attentional resources, possibly involving top-down brain mechanisms. The spontaneous mode, on the other hand, implies an unconscious process leading to what is reported as insights and results from effortless attention and possibly bottom-up brain processes.

We specifically tested the hypothesis that creative individuals are more prone to experience spontaneous pareidolia, which we defined as the ratio of the number of trials with RTs below 2 seconds to the total number of trials where pareidolia was reported. Our findings revealed a significant positive correlation between this metric of spontaneous pareidolia and creativity. Applied to the perspective of divergent perception, spontaneous pareidolia would correspond to the emergence of percepts in a context of low cognitive load, possibly as the result of an implicit resolution of confusion (67). This particularity of effortlessness associated with spontaneous creativity suggests that participants with higher spontaneous pareidolia might engage in the task with an effortless mode of attention. On the contrary, low-creative individuals may have more heavily relied on a deliberate mode of pareidolia, which implies a trial-and-error search process and higher cognitive load.

\section{Divergent perception}

Aside from proposing a functional link between creativity and perception of ambiguous stimuli, this study introduced a newly designed pareidolia task as a measure of divergent perception. We may think of the idea of divergent perception as the perceptual counterpart of divergent thinking. Hence, without having a direct "productive" outcome, pareidolia enables to account for a phenomenon of multiple coexisting solutions within a same problem space, as it is the case with classical divergent thinking tasks such as the Alternative Uses Task (AUT). The present study showed that creative individuals, as measured by three different questionnaires of creativity, have higher levels of fluency (number of objects) and flexibility (range of fractal dimensions) when performing a visual pareidolia task.

One might ask whether the perceptual processes investigated with a pareidolia task could merely be interpreted as imagination? A pareidolia task allows for divergent perception, which may overlap with processes generally involved in imagination, but is more specific to the case of finding multiple alternative solutions (here constructing multiple percepts) to a problem, as in divergent thinking. Arguably, creativity and imagination are intrinsically related and different types of imagination might not promote equally the emergence of creative idea generation. Several authors have drawn distinctions between categories of imagination, such as distinguishing between bottom-up and top-down imagination (68), or between mental imagery, novel-combinatorial (counterfactual), altered state and phenomenology-based imagination (69). Interestingly, pareidolia seems to encompass different categories of imagination, in that it relies both on the phenomenology associated with sensory experience and on the counterfactual process involved in "seeing" what is not objectively there based on prior experiences. Hence, we argue that divergent perception is a form of imagination that leads to idea generation through a balance between bottom-up and top-down processes.

\section{Pareidolia as a source of creative ideation}

The role of remote associations in creative processes has periodically appeared in the neuroscientific literature of creativity (70-72). Creative individuals, who are characterized by a 
propensity to perform remote associations, may use this skill at a perceptual level in order to more easily identify internal representations in ambiguous sensory information. Ambiguous stimuli would therefore constitute opportunities for a creative mind to apply top-down modulations that may result in conceptual expansion. By enriching sensory experience through a search for visual complexity, interactions with ambiguous stimuli afford the opportunity to resolve sensory dissonance with active top-down modulation. These top-down modulations allow the integration of sensory information into new conceptions and refined models. Hence, this adaptive strategy of making sense of (cognitively integrating) ambiguous sensory information gives rise to opportunities for constructing new models of the external world, based on the inherent complexity of stimuli. These new models are the very soil in which creativity plants its root and where new ideas can grow. Top-down modulations of ambiguous information enable internal representations to interact with each other within emergent perceptual content. Moreover, we might speculate that the inherent complexity of the stimuli enables the addition of uncertainty (natural noise) in the interaction between these internal representations. The malleability of internal representations, coupled with the inherent noise of sensory information, facilitates novel combinations of remote concepts, and the conjuration of seemingly non-familiar percepts. Hence, we suggest that pareidolia could be a source of creative ideation.

Numerous artists anecdotally reported experiencing pareidolia as a source of inspiration in their creative work. As exposed in (73) work Potential Images, Piero di Cosimo inspired his painting through looking at the sky, Novalis wrote about figures he saw in the clouds, and Chinese painter Sung Ti used the technique of looking at a dilapidated wall covered by a thin piece of white silk: "You gaze at it until you can see the ruins through the silk, its prominences, its levels, its zig-zags and its cleavages, storing them up in your mind, and fixing them in your eyes. Soon you will see men, birds, plants and trees, flying and moving among them. You may then ply your brush according to your fancy. (73)."

Leonardo Da Vinci recommended in his Treatise on Painting to look at rock formations, stained surfaces, ashes and clouds, to get inspiration: "Moreover, you can see various battles, and rapid actions of figures, strange expressions on faces, costumes, and an infinite number of things, which you can reduce to good, integrated form. (74)." By looking at these natural sceneries, DaVinci (74) demonstrates how prototypic internal representations can coexist within ambiguous sensory information and give rise to new modes of interactions, pointing to the natural role of fractal noise in the generation of new ideas. All these reports point to a functional link between pareidolia and creative inspiration, a link that is substantiated by the present study.

\section{Conclusion}

The present study is a first attempt to empirically link creativity and perceptual processes, using a pareidolia task. We showed that levels of creativity, whether measured by a questionnaire on phenomenological aspects of creative experience or through assessment of divergent thinking, significantly predict the occurrence of pareidolic experiences. We further identified systematic relations between pareidolia, creativity and the fractal dimension of the perceived visual stimuli. For high-contrast images, the propensity to experience pareidolia was higher in creative individuals specifically for low and high fractal dimensions. Taken together, these results indicate that (1) high levels of creativity are associated with enhanced pareidolia and (2) both fractal dimension and image contrast are key stimulus properties to manipulate when investigating pareidolic perception. These results also suggest that pareidolia could be a marker of idea generation and a predictor of creativity as it involves the perceptual ability of creating new ideas from the integration of ambiguous stimuli. Our findings call for future research to expand our understanding of the neuro-cognitive mechanisms associated with multiple dimensions of creativity, as well as the efficient encoding of image statistics (75). Future work might also benefit from integrating measures of visual imagery vividness (76) and content of the pareidolic percepts. The present results point towards the promise of probing creativity through the exploration of the neural dynamics associated with pareidolia. While a few studies have examined face pareidolia 
detection $(38,77,78)$, none have used fractal visual stimuli with open-ended designs. Another promising avenue for further research, is designing methods to measure the properties of pareidolic percepts (e.g. richness, diversity) in order to strengthen the theoretical link between creativity and pareidolia.

\section{Materials and Methods}

Participants. 50 neurotypical individuals (19 females) between the ages of 19 and $35(M=27.4$, $S D=3.24)$ took part in the experiment. All participants signed an informed consent form. Reaction time was not collected for two participants, leaving 48 participants for the analyses which required reaction times. The research project received ethics committee approval before all participants provided written informed consent to participate in this study.

Experimental protocol. In the first part of the experiment, 360 stimuli ( 3 levels of contrast and 12 levels of fractal dimension ranging from 0.8 to 1.9) were presented to each participant in a pseudo-random order. The participants faced the screen for the duration of the task and gave their answers using a standard keyboard. The images were of a size covering approximately 10 degrees of visual angle. The task of the participants was to detect the maximum number of percepts during the 8-second presentation of each stimulus. The participants had to press the spacebar when they perceived a first figurative object in the stimulus. After each stimulus, they reported the total number of perceived objects (scale from 0 to 5 -and-above). A block of 10 trials preceded the experiment so that the participants could get used to the task. Participants were told before beginning the task that at the end of the experiment, images for which they report the high number of objects will be presented to them again to assess the reported content. This procedure was deliberately implemented to reduce the chances that participants provide false responses during the test. During the experimental procedure, there was no mention of the term "creativity", as we wanted to avoid putting any pressure on the participants to perform well on a "creativity test". The participants were told that it was a study on perception, and that it could be seen as a game, similar to finding objects in the clouds and that there were no good or bad answers. The experimenter stayed with the participants during this test block in order to answer any questions. The experiment lasted about 60 minutes, divided into three blocks of twenty minutes, between which it was proposed to the participants to take a short break if desired.

Creativity assessment. The participants completed the Experience of Creativity Questionnaire (ECQ), a phenomenological measure of creativity, which is divided into two parts. The first part, which refers to a specific creative activity, comprises five subscales: power/pleasure, absorption, distinct experience, anxiety and clarity of preparation. The second part, reflecting the engagement with creative activities in general, is divided in three subscales: transformation, centrality of the experience, and transpersonal. The five subscales of the first part closely relate to flow-type experiences, which have been identified as a specific form of creativity (6). As a complementary measure to the ECQ, we chose a recently introduced behavioral measure of creativity, the Divergent Association Task (DAT), for which the data was collected in a follow-up study in 41 out of the 50 participants. Participants had to provide the ten most semantically distant words. Semantic distance between each pair of words was computed with GloVe (79) a freely available model which was pre-trained on the Common Crawl corpus, containing text from billions of web pages. Since the participants were French speakers, the data were collected in French. We tried using GloVe with a French database but found that a significant proportion of words were not recognized by the algorithm. Hence, the words were translated to English before computing the score. One of these participants was treated as an outlier as their score exceeded 3 SDs above the mean. The creativity tests were always administered after the pareidolia task, alongside a series of other tests (meditation, personality, flow state)

Stimulus design. Fractal dimension $(F D)$ describes the fractal scaling relation between the patterns observed at different magnifications (65). Many algorithms allow for the computation of fractal dimension (80). Images of lower $F D$ can be considered less detailed, while images of 
higher FD are more detailed and inherently complex (81-83). Fractals themselves are characterized as either exact or statistical. Exact fractals exhibit a geometry that repeats itself exactly at different levels of magnification, while statistical fractals contain a certain degree of randomness within their structure, leading to partial similarities between different magnifications.

Statistical fractal images were generated using a $1 / \mathrm{f}$ spatial noise (84) based on the inverse discrete Fourier Transform (Fig. 1), allowing control over the $F D$ of each image. The fractal dimension was derived from the spectral slope (Beta) of the distribution with the formula $F D=($ Beta * $2-6) / 2$. Beta values ranged from $-2.2(\approx$ brown noise) to -1.1 ( $\approx$ pink noise $)$ and corresponding $F D$ values from 0.8 to 1.9 . Variability in spectral slope between different images has also been demonstrated using natural images (85). This algorithm allowed to generate images with autocorrelated structure and values following a gaussian distribution. All images were of size 512 by 512 pixels. The images were manipulated to create three distinct levels of contrast. At high contrast, half of all image pixels were set to black and the other half to white by thresholding the grayscale image at the mean luminance level, as in (50). Two other contrast levels were generated by thresholding at $35 \%$ from each extreme of the spectrum for medium contrast (leaving $30 \%$ of pixels as grayscale), and at $20 \%$ from each extreme for low contrast (leaving $60 \%$ grayscale). The averaged root mean squared (RMS) values, which correspond to the standard deviation of the pixel intensities (86), were computed for each contrast level. RMS scores were $122.2(S D=4.7), 92.35(S D=8.5)$, and $65.7(S D=10.5)$ for high, mid and low contrast respectively. According to (50), the $F D$ is not affected by changing the contrast of the image. We validated that the $F D$ did not differ between contrast levels by applying the differential box-counting method (87), which allows to compute $F D$ on grayscale images.

Pareidolia measures. Pareidolia was assessed based on three indicators: reaction time $(R T)$, pareidolia occurrence (Par) and number of objects perceived ( $N$ _obj). $R T$ corresponds to the time between stimulus onset and first pareidolic perception. Pareidolia (Par) is a value between 0 and 1 representing the proportion of trials in which one or more pareidolic perceptions occurred. Par thus aims to capture participants' flexibility, defined as the capacity to experience pareidolia in a wide range of stimuli. Number of objects $\left(N \_o b j\right)$ represents the average number of pareidolic percepts on trials during which pareidolia occurred, and aims to capture the fluency aspect of pareidolia, i.e. the capacity to fluently modulate perceptions within the same stimulus. Finally, a measure of spontaneous pareidolia (Spont_par) was derived by computing the proportion of trials where pareidolia was reported within the first two seconds, compared to the total number of trials where pareidolia was reported. Several threshold values $(1.5 / 2 / 2.5 \mathrm{sec})$ were tested to ensure that our results are consistent (see Fig S3 for more details). Spontaneous pareidolia serves as a complementary measure of reaction time designed to target the trials that are likely to reflect the spontaneous emergence of pareidolic percepts.

\section{Statistical analysis plan}

Initial analyses. To confirm that stimulus properties influence pareidolia, we examined the linear and quadratic relation between $F D$ and pareidolia with Pearson correlation, and performed a repeated-measure ANOVA to investigate the effect of contrast on pareidolia. We also ran image-based analyses to check whether there was some evidence for consistency across participants in their responses to identical stimuli. First, we computed the mean value of pareidolia occurrence and number of percepts reported for each single image. We then computed new means for the same variable but this time after randomly shuffling the provided responses across all stimuli (as a realization of a mean of random responses for each stimulus). Most importantly, we tested the differences between the distribution of the original pareidolia response data and 1000 randomized sets of responses using two-sample Kolmogorov-Smirnov tests.

Generalized Linear Mixed Effect Model. Generalized Linear Mixed Effects Models (GLMM) are regression models that allow using non-normally distributed dependent variables and including random effects to model variables from different nested levels (88). By incorporating the variability 
inherent to nested variables, GLMM is a family of statistical models that allows the modelling of cross-level interactions. In the present case, the first level corresponds to the trial level, comprising pareidolia variables, $F D$ and contrast, while the second level corresponds to the participant level, comprising questionnaire variables (creative phenomenology, Crea_pheno). Hence, the use of GLMM enables us to include all the inherent variance in the data, and investigate first- and second-level variabilities simultaneously within a single model.

Two predictors (Crea_pheno, FD) were centered around the grand mean, while contrast was transformed into a binary variable based on preliminary analyses. To do so, medium and low contrast images were treated together as opposed to high-contrast images. Random slopes were also included for $F D$ terms following the guideline from (89) that suggests including random slope for any within-unit factor. When the predicted variable was Par, a binomial distribution with a logit link function was specified to best fit the binary outcome, while when the predicted variable was N_obj, a zero-truncated negative binomial distribution was specified. For our third dependent variable, RT, a logarithmic link function was specified. To achieve a good fit to the data with a GLMM, successive models were constructed and compared with each other while the level of complexity was increased at each iteration. To quantify the superiority of one model over another, ANOVAs comparing the Akaike Information Criterion (AIC) of two models were computed (90).

Principal analyses. We first tested whether creativity measures were correlated with pareidolia (averaged across all trials for each participant) using Spearman correlation. We then ran a Generalized Linear Mixed-effect Model (GLMM) that models the moderation effects of contrast and $F D$ (both linear and quadratic terms) on creativity. To explore further the moderator effect of $F D$ on the relation between creativity and pareidolia, we (1) ran regression analyses to predict pareidolia from creativity scores for each $F D$ (Bonferroni corrected), (2) examined the quadratic relation between $F D$ and pareidolia for high- and low-creatives and (3) ran the GLMM replacing Crea_pheno by the measure of divergent thinking (see Supplementary Materials).

\section{Acknowledgments}

AB is supported by a Fonds de recherche du Québec - Société et culture doctoral grant. YH is supported by a Courtois Neuromod graduate fellowship. KJ is supported by funding from the Canada Research Chairs program and a Discovery Grant from the Natural Sciences and Engineering Research Council of Canada, a New Investigators Award from the Fonds de Recherche du Québec - Nature et Technologies and an IVADO- Apogée fundamental research project grant. Finally, AB would also like to acknowledge the support of his mother, who taught him that believing was the deepest strength, and who exposed him to a broad repertoire of creative mediums. 


\section{References}

1. Sternberg, R. J., \& Lubart, T. I. (1999). The concept of creativity: Prospects and paradigms. Handbook of creativity, 1, 3-15.

2. Barbot, B., Besançon, M., \& Lubart, T. (2015). Creative potential in educational settings: Its nature, measure, and nurture. Education 3-13, 43(4), 371-381. https://doi.org/10.1080/03004279.2015.1020643

3. Dietrich, A. (2007). Who's afraid of a cognitive neuroscience of creativity? Methods, 42(1), 22-27.

4. Fryer, M. (2012). Some key issues in creativity research and evaluation as seen from a psychological perspective. Creativity Research Journal, 24(1), 21-28. https://doi.org/10.1080/10400419.2012.649236

5. Glăveanu, V. P. (2014b). The psychology of creativity: A critical reading. Creativity: Theories - $\quad$ Research - Applications, 1(1), 10-32. https://doi.org/10.15290/ctra.2014.01.01.02

6. Dietrich, A. (2004). The cognitive neuroscience of creativity. Psychonomic bulletin \& review, 11(6), 1011-1026.

7. Jung, R. E., Gasparovic, C., Chavez, R. S., Flores, R. A., Smith, S. M., Caprihan, A., \& Yeo, R. A. (2009). Biochemical support for the "threshold" theory of creativity: a magnetic resonance spectroscopy study. Journal of Neuroscience, 29(16), 5319-5325.

8. Simonton, D. K. (2010). Creative thought as blind-variation and selective-retention: Combinatorial models of exceptional creativity. Physics of life reviews, 7(2), 156-179.

9. Wiggins, G. A. (2006). Searching for computational creativity. New Generation Computing, 24(3), 209-222.

10. Dietrich, A., \& Kanso, R. (2010). A review of EEG, ERP, and neuroimaging studies of creativity and insight. Psychological bulletin, 136(5), 822.

11. Sawyer, R. K. (2011). Explaining creativity: The science of human innovation. Oxford university press.

12. Guilford, J. P. (1950). Creativity. American Psychologist, 5, 444-454. doi:10.1037/h0063487

13. Weisberg, R. W. (2006). Creativity: Understanding innovation in problem solving, science, invention, and the arts. John Wiley \& Sons.

14. Guilford, J. P. (1967). The nature of human intelligence. New York, NY: McGraw-Hill.

15. Kim, K. H. (2006). Can we trust creativity tests? A review of the Torrance Tests of Creative Thinking (TTCT). Creativity research journal, 18(1), 3-14.

16. Dietrich, A. (2019). Types of creativity. Psychonomic bulletin \& review, 26(1), 1-12. 
17. Dietrich, A. (2019). Where in the brain is creativity: a brief account of a wild-goose chase. Current Opinion in Behavioral Sciences, 27, 36-39.

18. Simonton, D. K. (2015). On praising convergent thinking: Creativity as blind variation and selective retention. Creativity Research Journal, 27(3), 262-270.

19. Ward, T. B., Smith, S. M., \& Finke, R. A. (1999). Creative cognition. Handbook of creativity, 189, 212.

20. Heath, D., \& Ventura, D. (2016, June). Before a computer can draw, it must first learn to see. In Proceedings of the 7th international conference on computational creativity (pp. 172-179).

21. Flowers, J. H., \& Garbin, C. P. (1989). Creativity and perception. In Handbook of creativity (pp. 147-162). Springer, Boston, MA.

22. Berns, G. (2008). Neuroscience sheds new light on creativity. Fast Company, (129).

23. Malinin, L. H. (2019). How Radical is Embodied Creativity?. Frontiers in psychology, 10, 2372.

24. D'Angelo, D. (2019). The phenomenology of embodied attention. Phenomenology and the Cognitive Sciences, 1-18.

25. Fischer R, Scheib J (1971) Creative performance and the hallucinogenic drug-induced creativeexperience or one man's brain-damage is another's creativity. Confin Psychiatr 14:174-202

26. Nelson, C. B. (2005). The creative process: A phenomenological and psychometric investigation of artistic creativity (Doctoral dissertation).

27. Pereira, Á., \& Tschimmel, K. (2012). The Design of Narrative Jewelry as a Perception in Action Process. In DS 73-1 Proceedings of the 2nd International Conference on Design Creativity Volume 1.

28. Petchkovsky, L. (2008). Some Preliminary Reflections on the Biological Substrate of Meaning-Making. In The Uses of subjective experience. Proceedings of the Conference 'The Uses of Subjective Experience: A Weekend of Conversations between ANZSJA Analysts and Academics who Work with Jung's Ideas (pp. 20-21).

29. Fyfe, S., Williams, C., Mason, O. J., \& Pickup, G. J. (2008). Apophenia, theory of mind and schizotypy: Perceiving meaning and intentionality in randomness. Cortex, 44(10), 1316-1325.

30. Meschiari, M. (2009). Roots of the savage mind. Apophenia and imagination as cognitive process. Quaderni di semantica: rivista internazionale di semantica teorica e applicata, 30(2), 183-262.

31. Barrett, J. L. (2000). Exploring the natural foundations of religion. Trends in cognitive sciences, 4(1), 29-34.

32. Kapur, S. (2003). Psychosis as a state of aberrant salience: a framework linking biology, phenomenology, and pharmacology in schizophrenia. American journal of Psychiatry, 160(1), 13-23. 
33. Kapur, S., Mizrahi, R., \& Li, M. (2005). From dopamine to salience to psychosis-linking biology, pharmacology and phenomenology of psychosis. Schizophrenia research, 79(1), 59-68.

34. Vercammen, A., de Haan, E. H., \& Aleman, A. (2008). Hearing a voice in the noise: auditory hallucinations and speech perception. Psychological medicine, 38(8), 1177-1184.

35. Yokoi, K., Nishio, Y., Uchiyama, M., Shimomura, T., lizuka, O., \& Mori, E. (2014). Hallucinators find meaning in noises: pareidolic illusions in dementia with Lewy bodies. Neuropsychologia, 56, 245-254.

36. Belayachi, S., Laloyaux, J., Larø i, F., \& Van der Linden, M. (2015). Internal encoding style and schizotypy: toward a conceptually driven account of positive symptoms. Journal of personality disorders, 29(3), 303-315.

37. Hong, K., Chalup, S. K., King, R. A., \& Ostwald, M. J. (2013, April). Scene perception using pareidolia of faces and expressions of emotion. In 2013 IEEE Symposium on Computational Intelligence for Creativity and Affective Computing (CICAC) (pp. 79-86). IEEE.

38. Liu, J., Li, J., Feng, L., Li, L., Tian, J., \& Lee, K. (2014). Seeing Jesus in toast: neural and behavioral correlates of face pareidolia. Cortex, 53, 60-77.

39. Lee, J. (2016). I See Faces: Popular pareidolia and the proliferation of meaning.

40. Diana, L., Frei, M., Chesham, A., de Jong, D., Chiffi, K., Nyffeler, T., ... \& Müri, R. M. (2021). A divergent approach to pareidolias-Exploring creativity in a novel way. Psychology of aesthetics, creativity, and the arts.

41. Rogowitz, B. E., \& Voss, R. (1990, October). Shape perception and low-dimension fractal boundary contours. In Human Vision and Electronic Imaging: Models, Methods, and Applications (Vol. 1249, pp. 387-394). International Society for Optics and Photonics.

42. Bies, A. J., Kikumoto, A., Boydston, C., Greenfield, A., Chauvin, K. A., Taylor, R. P., \& Sereno, M. E. (2016, August). Percepts from noise patterns: The role of fractal dimension in object pareidolia. In Vision Science Annual Meeting.

43. Taylor, R. P., Martin, T. P., Montgomery, R. D., Smith, J. H., Micolich, A. P., Boydston, C., ... \& Spehar, B. (2017). Seeing shapes in seemingly random spatial patterns: Fractal analysis of Rorschach inkblots. PloS one, 12(2), e0171289.

44. Almeida, L. S., Prieto, L. P., Ferrando, M., Oliveira, E., \& Ferrándiz, C. (2008). Torrance Test of Creative Thinking: The question of its construct validity. Thinking skills and creativity, 3(1), 53-58.

45. Aks, D. J., \& Sprott, J. C. (1996). Quantifying aesthetic preference for chaotic patterns. Empirical studies of the arts, 14(1), 1-16.

46. Hagerhall, C. M., Purcell, T., \& Taylor, R. (2004). Fractal dimension of landscape silhouette outlines as a predictor of landscape preference. Journal of environmental psychology, 24(2), 247-255. 
47. Taylor, R. P., Spehar, B., Wise, J. A., Clifford, C. W., Newell, B. R., Hagerhall, C. M., ... \& Martin, T. P. (2005). Perceptual and physiological responses to the visual complexity of fractal patterns. Nonlinear Dynamics Psychol. Life. Sci, 9, 89-114.

48. Viengkham, C., \& Spehar, B. (2018). Preference for fractal-scaling properties across synthetic noise images and artworks. Frontiers in psychology, 9, 1439.

49. Taylor, R. P., \& Spehar, B. (2016). Fractal fluency: an intimate relationship between the brain and processing of fractal stimuli. In The fractal geometry of the brain (pp. 485-496). Springer, New York, NY.

50. Spehar, B., Walker, N., \& Taylor, R. P. (2016). Taxonomy of individual variations in aesthetic responses to fractal patterns. Frontiers in human neuroscience, 10, 350.

51. Nelson, B., \& Rawlings, D. (2009). How does it feel? The development of the experience of creativity questionnaire. Creativity Research Journal, 21(1), 43-53.

52. Olson, J. A., Nahas, J., Chmoulevitch, D., Cropper, S. J., \& Webb, M. E. (2021). Naming unrelated words predicts creativity. Proceedings of the National Academy of Sciences, 118(25).

53. Clark, T. S., \& Linzer, D. A. (2015). Should I use fixed or random effects. Political science research and methods, 3(2), 399-408.

54. Wagemans, J., Elder, J. H., Kubovy, M., Palmer, S. E., Peterson, M. A., Singh, M., \& von der Heydt, R. (2012). A century of Gestalt psychology in visual perception: I. Perceptual grouping and figure-ground organization. Psychological bulletin, 138(6), 1172.

55. Bergum, J. E., \& Bergum, B. O. (1979). Self-perceived creativity and ambiguous figure reversal rates. Bulletin of the Psychonomic Society, 14(5), 373-374.

56. Klintman, H. (1984). Original thinking and ambiguous figure reversal rates. Bulletin of the Psychonomic Society, 22(2), 129-131.

57. Wernery, J. (2013). Bistable perception of the Necker cube: In the context of cognition \& personality (Doctoral dissertation, ETH Zurich).

58. Benedek, M., Franz, F., Heene, M., \& Neubauer, A. C. (2012). Differential effects of cognitive inhibition and intelligence on creativity. Personality and individual differences, 53(4), 480-485.

59. Radel, R., Davranche, K., Fournier, M., \& Dietrich, A. (2015). The role of (dis) inhibition in creativity: Decreased inhibition improves idea generation. Cognition, 134, 110-120.

60. Nijstad, B. A., De Dreu, C. K., Rietzschel, E. F., \& Baas, M. (2010). The dual pathway to creativity model: Creative ideation as a function of flexibility and persistence. European Review of Social Psychology, 21(1), 34-77.

61. Dreu, C. K. D., Nijstad, B. A., \& Baas, M. (2011). Behavioral activation links to creativity because of increased cognitive flexibility. Social Psychological and Personality Science, 2(1), $72-80$.

62. Wu, X., Gu, X., \& Zhang, H. (2019). The facilitative effects of ambiguous figures on creative solution. The Journal of Creative Behavior, 53(1), 44-51. 
63. Richards, R. (2001). Millennium as opportunity: Chaos, creativity, and Guilford's Structure of Intellect Model. Creativity Research Journal, 13(3-4), 249-265.

64. Taylor, R. P., Micolich, A. P., \& Jonas, D. (1999). Fractal analysis of Pollock's drip paintings. Nature, 399(6735), 422-422.

65. Spehar, B., Clifford, C. W., Newell, B. R., \& Taylor, R. P. (2003). Universal aesthetic of fractals. Computers \& Graphics, 27(5), 813-820.

66. Roddy, S., \& Furlong, D. (2014). Embodied aesthetics in auditory display. Organised Sound, 19(1), 70-77.

67. Shen, W., Yuan, Y., Liu, C., \& Luo, J. (2016). In search of the 'Aha!' experience: Elucidating the emotionality of insight problem-solving. British Journal of Psychology, 107(2), 281-298.

68. Vyshedskiy, A. (2019). Neuroscience of imagination and implications for human evolution.

69. Abraham, A. (2016). The imaginative mind. Human Brain Mapping, 37(11), 4197-4211.

70. Mednick, S. (1962). The associative basis of the creative process. Psychological review, 69(3), 220.

71. Mednick, S. A. (1968). The remote associates test. The Journal of Creative Behavior, 2(3), 213-214.

72. Sassenberg, K., Moskowitz, G. B., Fetterman, A., \& Kessler, T. (2017). Priming creativity as a strategy to increase creative performance by facilitating the activation and use of remote associations. Journal of Experimental Social Psychology, 68, 128-138.

73. Gamboni, D. (2002). Potential images: Ambiguity and indeterminacy in modern art. Reaktion Books.

74. Da Vinci, L. (1956). Treatise on Painting, ed. AP McMahon, Princeton, 2.

75. Simoncelli, E. P., \& Olshausen, B. A. (2001). Natural image statistics and neural representation. Annual review of neuroscience, 24(1), 1193-1216.

76. Salge, J. H., Pollmann, S., \& Reeder, R. R. (2021). Anomalous visual experience is linked to perceptual uncertainty and visual imagery vividness. Psychological research, 85(5), 1848-1865.

77. Wardle, S. G., Taubert, J., Teichmann, L., \& Baker, C. I. (2020). Rapid and dynamic processing of face pareidolia in the human brain. Nature communications, 11(1), 1-14.

78. Rekow, D., Baudouin, J. Y., Brochard, R., Rossion, B., \& Leleu, A. (2021). Rapid neural categorization of facelike objects predicts the perceptual awareness of a face (face pareidolia). bioRxiv.

79. Pennington, J., Socher, R., \& Manning, C. D. (2014, October). Glove: Global vectors for word representation. In Proceedings of the 2014 conference on empirical methods in natural language processing (EMNLP) (pp. 1532-1543). 
80. Lopes, R., \& Betrouni, N. (2009). Fractal and multifractal analysis: a review. Medical image analysis, 13(4), 634-649.

81. Cutting, J. E., \& Garvin, J. J. (1987). Fractal curves and complexity. Perception \& Psychophysics, 42(4), 365-370.

82. Pentland, A. P. (1984). Fractal-based description of natural scenes. IEEE Transactions on Pattern Analysis \& Machine Intelligence, (6), 661-674.

83. Gilden, D. L., Schmuckler, M. A., \& Clayton, K. (1993). The perception of natural contour. Psychological review, 100(3), 460.

84. Lennon, J. J. (2000). Red-shifts and red herrings in geographical ecology. Ecography, 23(1), 101-113.

85. Tolhurst, D. J., Tadmor, Y., \& Chao, T. (1992). Amplitude spectra of natural images. Ophthalmic and Physiological Optics, 12(2), 229-232.

86. Peli, E. (1990). Contrast in complex images. JOSA A, 7(10), 2032-2040.

87. Chaudhuri, B. B., \& Sarkar, N. (1995). Texture segmentation using fractal dimension. IEEE Transactions on pattern analysis and machine intelligence, 17(1), 72-77.

88. Bates, D., Mächler, M., Bolker, B., \& Walker, S. (2014). Fitting linear mixed-effects models using Ime4. arXiv preprint arXiv:1406.5823.

89. Barr, D. J. (2013). Random effects structure for testing interactions in linear mixed-effects models. Frontiers in psychology, 4, 328.

90. Zuur, A., leno, E. N., Walker, N., Saveliev, A. A., \& Smith, G. M. (2009). Mixed effects models and extensions in ecology with $R$. Springer Science \& Business Media. 